\title{
Neonatology Today is Committed to Improving the Digital Experience of the Readers
}

Fu-Sheng Chou, MD Ph.D., Japmeet Sandhu OSM III, Mitchell Goldstein, MD

Since the Loma Linda Publishing Company and the faculty at the Division of Neonatology of Loma Linda University School of Medicine took over the production of Neonatology Today in March 2018 , there has been a steady increase in the number of articles in each issue, thanks to the expansion of the contracted column articles (Figure 1). We want to thank all the contributing authors for making Neonatology Today a sustainable publication.

"Six months ago, we developed a new website (https://neonatologytoday.org) intending to expand further the digital footprint of the Journal in this modern era of the digital world. The custom-designed web site was developed using the $R$ programming language and the Shiny package. (1)"

Six months ago, we developed a new website (https://neonatologytoday.org) intending to expand further the digital footprint of the Journal in this modern era of the digital world. The customdesigned web site was developed using the R programming language and the Shiny package. (1) In addition to providing the links to the digital (HTML) and the print (PDF) versions of the articles, we also added a Search function for readers to quickly find articles of interest. Moreover, we developed online manuscript submission and review systems, which have not been used by our authors and reviewers yet. The website was visited by readers like you from all over the world. The traffic came from all five continents over 34 countries (Figure 2). We share this information to let our readers and authors know that the information available in the Journal can reach every corner of the world. We hope that some of the distributed knowledge has made an impact on neonates around the world.

Neonatology Today is committed to bringing you, our loyal readers, the most timely and accurate information. In addition, we aspire to provide our practicing neonatologists and academic researchers with inspirations for your next quality improvement or research projects. To increase the Journal's visibility in academic society, we had the Journal registered with Crossref. Following this step is assigning a digital object identifier (DOI) to each HTML article and including metadata in the HTML files. As many of our readers who read academic journals are very aware, DOI is a unique web link that takes the readers to the article's web page. DOI can also import articles into the citation managers (Mendeley, Paperpile, EndNote, Zotero, etc.), allowing the metadata (year, volume, issue, page, title, abstract, etc.) to be populated automatically. In addition to assigning DOls to all future articles, we are also ac-

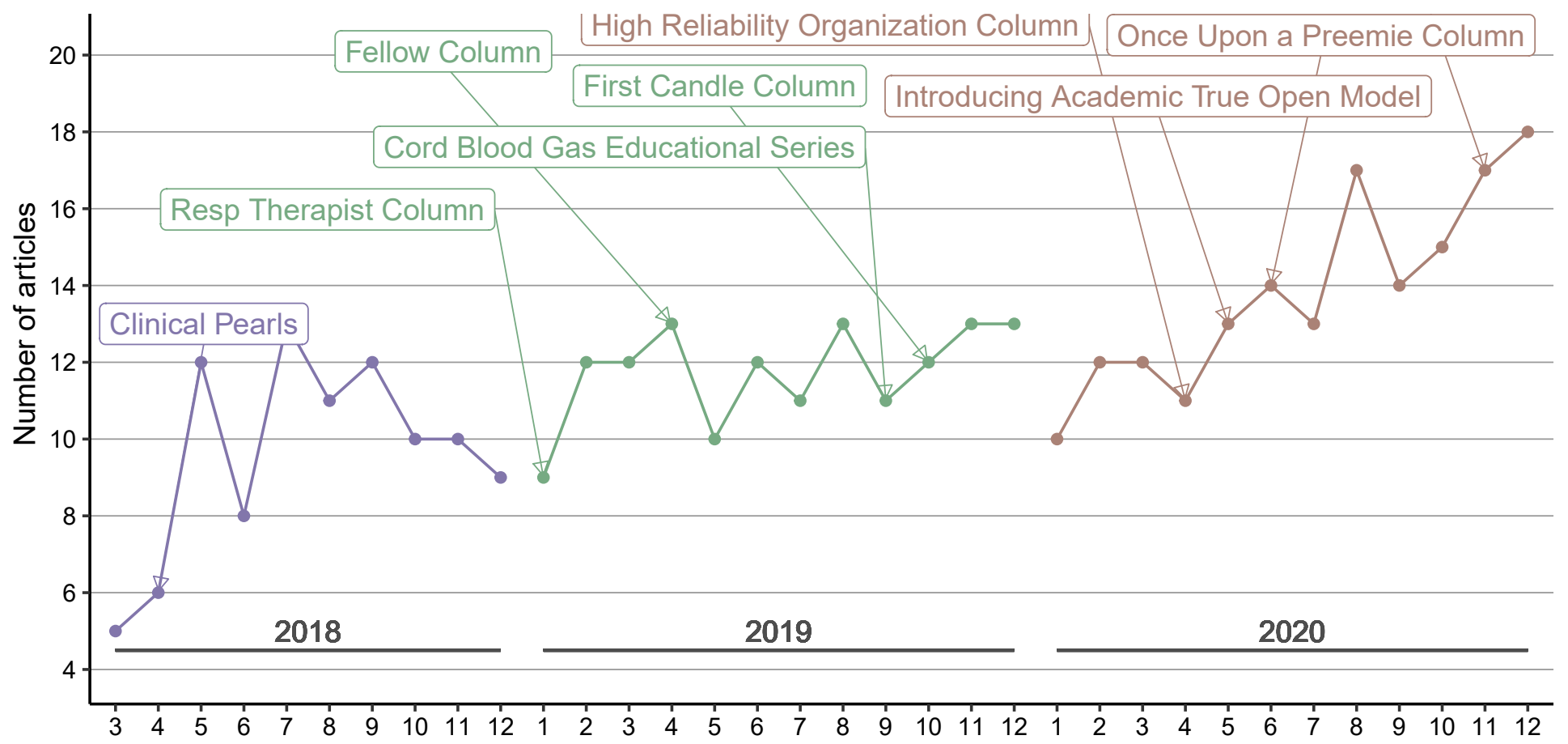

Month

Figure 1. 
tively working on assigning DOIs to the archived articles (work led by Japmeet Sandhu). With assigning the DOls to all articles, our goal is to make citations of articles published with Neonatology Today straightforward and meet the industry standards.

\section{"Our goal is to increase monthly} publications of articles by increasing the number of unsolicited manuscripts. If you have an interesting case to report, an interesting topic to review, any personal opinions or comments to make, or a research study to report, but you are not confident how to get started, feel free to reach out to the editorial team to discuss your goal."

Our goal is to increase monthly publications of articles by increasing the number of unsolicited manuscripts. If you have an interesting case to report, an interesting topic to review, any personal opinions or comments to make, or a research study to report, but you are not confident how to get started, feel free to reach out to the editorial team to discuss your goal. We are here not only to help you to accomplish your goal but to achieve your goal efficiently.

Please visit the article HTML pages and enter the DOls into your citation manager to start a new collection with Neonatology Today's articles. We also welcome you to try out our new online manuscript submission system, with which you as the author will only focus on the content of the manuscript but not the formatting part of it. An instruction manual for the online manuscript submission system is currently in production. A separate announcement will be made when the manual is available to the public.

Keep asking questions and keep writing!

Reference:

1. Chou F-S. An unexpected use of the shiny package for $R$. Neonatology Today. 2020;15(11):26-8.

Disclosure: The authors have no disclosures.

\section{NT}

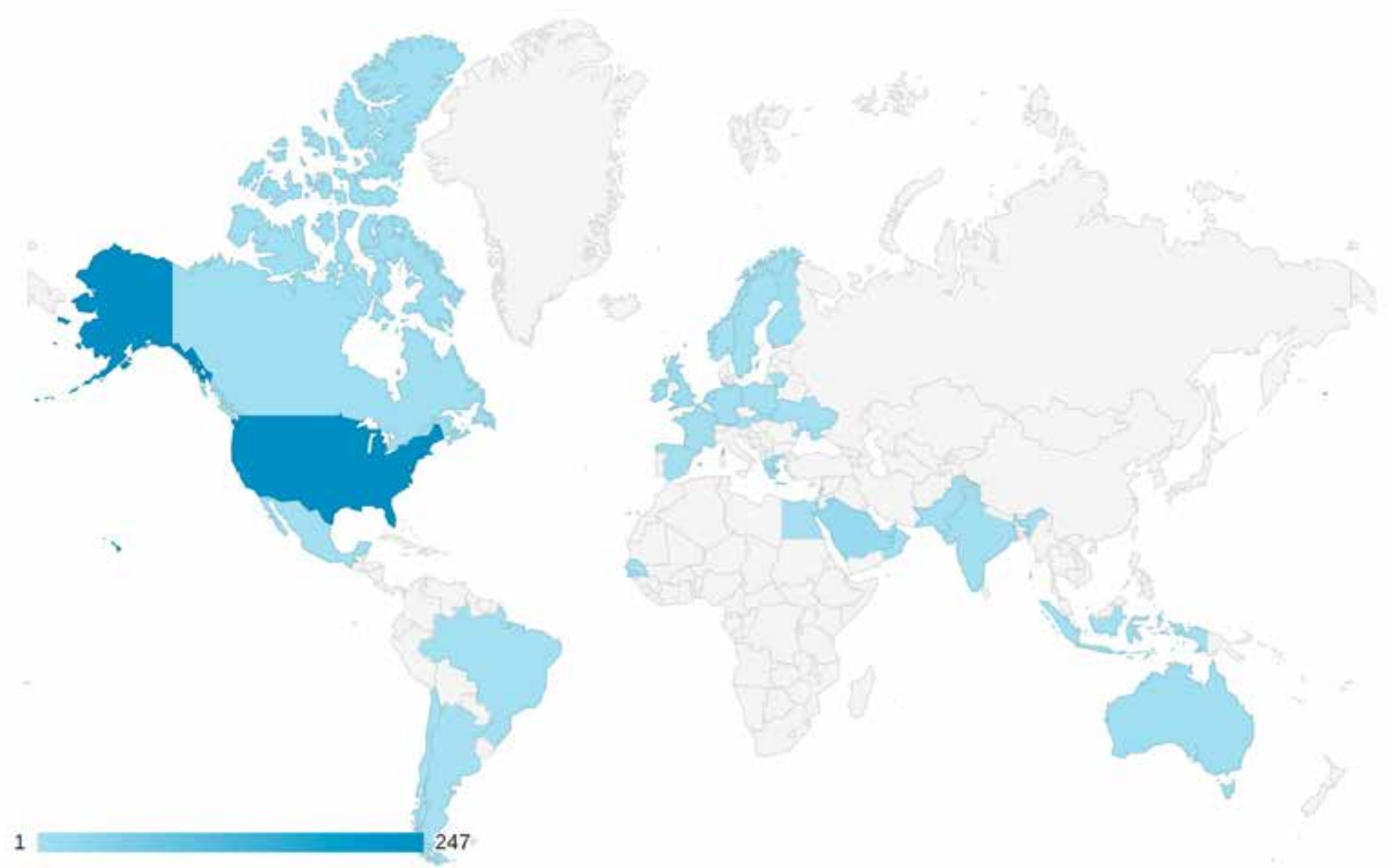

Figure 2. 
Corresponding Author

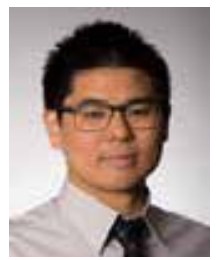

Fu-Sheng Chou, MD, PhD -

Senior Associate Editor,

Director, Digital Enterprise

Neonatology Today

Assistant Professor of Pediatrics

Division of Neonatology, Department of Pediatrics

Loma Linda University Children's Hospital

FChou@llu.edu

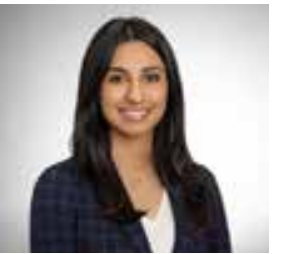

Japmeet Sandhu, OMS III

Western University of Health Sciences

Pomona, CA

Email: Japmeet Sandhu <japmeet.sandhu@westernu.edu>

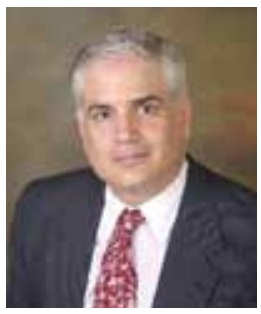

Mitchell Goldstein, MD

Professor of Pediatrics

Loma Linda University School of Medicine

Division of Neonatology

Department of Pediatrics

mgoldstein@llu.edu

\section{Still a Preemie?}

Some preemies are born months early, at extremely low birthweights. They fight for each breath and face nearly

insurmountable health obstacles.

But that's not every preemie's story.

Born between 34 and 36 weeks gestation?

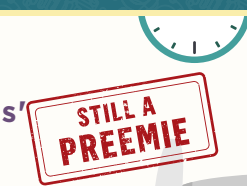

Just like preemies born much earlier, these "late preterm" infants can face:
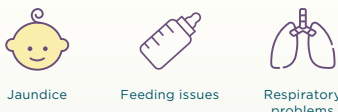

Respiratory
problems

And their parents, like all parents

of preemies, are at risk for

postpartum depression and PTSD.

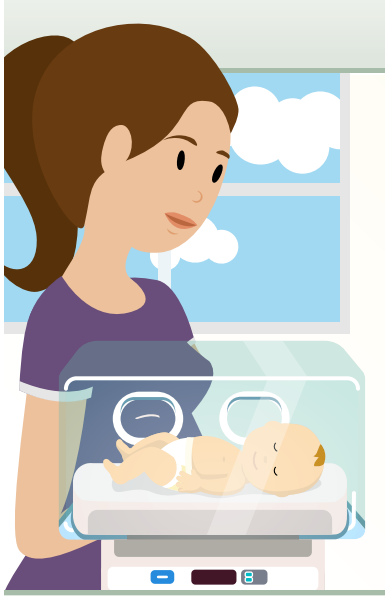

Born preterm

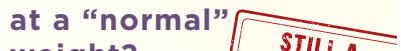
weight?

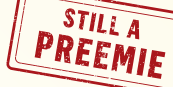

Though these babies look healthy, they can still have complications and require NICU care.

But because some health plans determine coverage based on a preemie's weight, families of babies that weigh more may

face access barriers and

unmanageable medical bills.
Born preterm but not admitted to the NICU?

Even if preterm babies don't require NICU care, they can still face health challenges. Those challenges can extend through childhood, adolescence and even into adulthood.
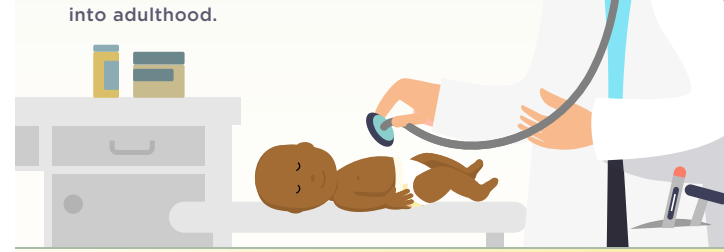

Some Preemies

Will spend weeks

in. in the hospital

9- Will have lifelong

우요 Are disadvantaged from birth
All Preemies

Face health

\$) risks

$\oplus$ Deserve appropriate

health coverag

Qo Need access to

$N C f I H$ National Coalition

www.infanthealth.org 